\title{
Comparison of single- and multi-joint lower body resistance training upon strength increases in recreationally active males and females: a within-participant unilateral training study
}

\author{
Alexander Goncalves (1), Paulo Gentil (2), James Steele (1,3), Jürgen Giessing (4), \\ Antonio Paoli (5), James P. Fisher (1)
}

(1) School of Sport, Health and Social Science, Southampton Solent University, UK; (2) Faculty of Physical Education and Dance, Federal University of Goias, Brazil; (3) ukactive Research Institute, London, UK; (4) University of Koblenz-Landau, Germany; (5) University of Padova, Italy

This article is distributed under the terms of the Creative Commons Attribution Noncommercial License (CC BY-NC 4.0) which permits any noncommercial use, distribution, and reproduction in any medium, provided the original author(s) and source are credited.

\begin{abstract}
The present study compared strength increases resulting from either single-joint (SJ) or multijoint (MJ) lower body resistance exercise. A within-participants design was utilised. Ten recreationally active participants (males; $n=5$, and females; $n=5$ ) had their lower limbs randomly allocated to perform both unilateral MJ (leg press; LP) and unilateral SJ (knee extension; KE, and seated knee flexion; KF) exercises. Participants trained 2 d.week-1 for 6 weeks. Pre- and post-intervention maximal strength (1-repetition maximum; 1RM) was measured for leg press, knee extension, and seated knee flexion exercises. Statistically significant strength increases occurred for both SJ and MJ groups, with significantly greater increases in 1RM for the MJ compared to the SJ group ( $\mathrm{p}<0.001$ for all exercises). This study supports the use of MJ exercise for strength increases across lower body MJ and SJ movements, suggesting a time-efficient, and simple approach to resistance training may be efficacious. This might serve to promote greater adherence in the lay population as well as serving useful for rehabilitation professionals and strength and conditioning coaches in managing resistance exercise around skill/sport specific training.
\end{abstract}

Key Words: compound exercise, isolation exercise, exercise selection.

Eur J Transl Myol 29 (1): 14-21, 2019

Resistance training (RT) is generally accepted as the primary method of increasing muscular strength and size. Indeed, muscle mass ${ }^{1}$ and muscular strength $^{2}$ are evidenced predictors of longevity and reduction in allcause mortality, and it has been argued RT should hold a more prominent place within public health recommendations regarding exercise. ${ }^{3}$ Moreover, RT has been suggested as a valuable tool for preventing and treating sports injury. ${ }^{4,5}$ However, RT adherence remains low. ${ }^{6}$ With this in mind, a growing body of research has considered more time-efficient approaches of reduced volume RT, for example single-set protocols of 10 exercises lasting only 20 minutes per session, ${ }^{7}$ or even fewer - where a full-body workout lasted 12-15 minutes and consisted of only 5 multi-joint (MJ) exercises (pulldown, chest press, seated row, overhead press and leg press) ${ }^{8}$. Indeed, a number of studies have compared the use of single-joint (SJ) or isolation exercises involving a muscle or muscle group acting at a single joint to produce rotary movement/torque (e.g. elbow flexion/extension, knee flexion/extension, etc.), to $\mathrm{MJ}$ or compound exercises involving multiple muscles acting at multiple joints to produce force in a linear output. Examples are a leg press, where movement occurs at the knee and hip joints, and a chest press, seated row, and pull-down exercises, e. where movement occurs at both the elbow and shoulder joints. For example, Gentil, et al. ${ }^{9}$ compared a group of previously untrained males performing MJ exercises (bench press and pull-down) to a group performing $\mathrm{MJ}+\mathrm{SJ}$ exercises for 10 weeks. Elbow flexor peak torque statistically significantly improved in both groups with no between group differences. In a further study, this time considering trained males, de França et al. ${ }^{10}$ considered the effects of adding SJ exercises to a 10week, upper-body MJ program. The MJ group performed 5 pressing exercises for shoulders and chest (Monday and Thursday) and 5 pulling exercises (Tuesday and Friday), 
whilst the MJ+SJ group performed 2 additional exercises for the elbow extensors (Monday and Thursday) and elbow flexors (Tuesday and Friday). Their findings indicated that both $\mathrm{MJ}$ and $\mathrm{MJ}+\mathrm{SJ}$ participants significantly increased elbow flexion and extension 1repetition maximum (RM) with no between group differences. Finally, the most recent publication comparing $\mathrm{MJ}$ and $\mathrm{MJ}+\mathrm{SJ}$ resistance training reported significant increases in 10RM for elbow flexion, elbow extension and knee extension exercises, with no between group differences, in untrained females after an 8-week RT program. ${ }^{11}$ However, the MJ+SJ group showed significantly greater increases in flexed arm circumference compared to the $\mathrm{MJ}$ group $(\mathrm{MJ}+\mathrm{SJ}=1.22$ $\pm 0.05 \mathrm{~cm}$ vs. $\mathrm{MJ}=0.93 \pm 0.05 \mathrm{~cm}, p=0.002)$. Similar results have been reported when comparing MJ (pulldown) and SJ (elbow flexion) exercises only (and thus matched volume for the elbow flexor muscle group). Gentil, Soares, and Bottaro ${ }^{12}$ reported that both MJ and SJ groups increased elbow flexor peak torque to a similar extent having performed a RT intervention for 10 weeks. More recently, Paoli, Gentil, Moro, et al. ${ }^{13}$ compared amateur soccer players with no RT experience performing either a SJ- or MJ- RT protocol for 8 weeks. The authors reported significantly greater $(p<0.05)$ increases in $1 \mathrm{RM}$ bench press $(\mathrm{MJ}=10.9 \%, \mathrm{SJ}=8.1 \%)$, $1 \mathrm{RM}$ knee extension $(\mathrm{MJ}=18.9 \%, \mathrm{SJ}=12.4 \%)$, and $1 \mathrm{RM}$ back squat $(\mathrm{MJ}=13.8 \%, \mathrm{SJ}=8.3 \%)$ exercises in favour of the MJ group. As the authors suggest, the greater increases in strength for the MJ group might be a product of improved skill as a result of practicing the motor schema for the MJ bench press and back squat exercises which involve more complex movement patterning (i.e. in essence practicing the test ${ }^{14}$ ). However, the favourable results for the KE exercise are not supported by exercise specificity, suggesting that neural, mechanical and/or morphological adaptations for maximal strength were more favourable in the MJ group. This might be a product of greater muscle damage and delayed recovery as a result of the SJ exercises which may influence preparedness to train. Soares, et al. ${ }^{15}$ compared acute responses in isometric peak torque in both SJ (biceps preacher curl) and MJ (seated row) exercises using a unilateral, within-participant design. Analyses revealed that the decrease in peak torque was greater immediately after exercise $(-26.8 \%$ vs. $-15.1 \%)$ and remained lower than baseline $(-8.4 \%)$ at 24 hours post-exercise in the SJ compared to the MJ group. To date much of the literature has considered only upper body exercises, ${ }^{9,10}$ and none have considered knee flexion strength. Hamstring strength is particularly important for injury rehabilitation and prevention. ${ }^{16,17}$ Poor knee flexion strength has been associated with injury risk, ${ }^{4,5}$ and regaining quadriceps and hamstring muscle strength are important rehabilitation goals. ${ }^{18,19}$ However, we should question whether this is best done using SJ and/or MJ exercises, when, during MJ exercises, biarticular muscles shorten at one joint whilst lengthening at the other joint (e.g. the hamstrings during lower-body MJ exercises). Confirming this, previous studies show that the electromyographically measured activity of the hamstrings is limited in exercises such as the leg press. ${ }^{20,21}$ Therefore, it is debatable if the use of lower body MJ exercises would improve knee flexion strength and, if so, if the effects would be similar to those achieved by SJ exercises. Another important point is that none of the intervention studies have considered a within participant's design which controls for some of the individual variables which might also impact RT adaptations (e.g. sleep, nutrition, hormone levels, stress levels, etc.) thus reducing between intervention variability due to these factors to increase the informativeness of comparisons. ${ }^{22}$ With the above in mind, the present study's aims were to compare increases in strength when performing either SJ or MJ lower body exercises using unilateral movements in recreationally active males and females.

\section{Materials and Methods}

\section{Study Design}

Due to difficulty in recruitment for training intervention studies, and to increase study informativeness, we utilised a within-participants experimental design in order to increase power and precision for comparisons in small sample sizes. ${ }^{22}$ Unilateral MJ (leg press) exercise was performed on one leg and unilateral SJ (knee extension and seated knee flexion) exercise performed on the contralateral leg. Participants were randomised and counterbalanced to perform either the MJ (and thus also the SJ) exercise on either their dominant, or nondominant leg. Unilateral RT was performed $2 \mathrm{~d} \cdot$ week $^{-1}$ over the course of 6 weeks and outcome measures included changes in strength for both MJ and SJ lower body exercises.

\section{Participants}

Following approval from the relevant ethics committee (in accordance with the Helsinki Declaration of 1975; ethics code: HESS\#858), ten recreationally active males $(n=5)$ and females $(n=5)$ were recruited (see Table 1 for participant characteristics). Sample size was determined based upon precision of $f=0.5$ (i.e. margin of error, or half width of $95 \%$ confidence intervals, to have on average expected precision of 0.5 standard deviation units) for changes in strength in paired comparisons. Paired comparison correlation for this precision analysis was based upon prior data from within participant studies in our laboratory showing an $r=0.88$ for paired changes in strength. ${ }^{23,24}$ Sample estimate was determined using Exploratory Software for Confidence Intervals (ESCI ${ }^{25}$ and suggested $N=7$ using $t$ where the population $\sigma$ is unknown. With $99 \%$ assurance suggested sample size was $N=12$; however, recruitment was conducted during a single semester at our institution and only 10 participants volunteered. ESCI thus indicated that with $N=10$ assurance was $96 \%$. All participants were aged 


\begin{tabular}{ccc}
\multicolumn{3}{c}{ Table 1. Participant Demographics $($ mean \pm SD) } \\
\hline Variable & Males $(n=5)$ & Females $(n=5)$ \\
\hline Age (years) & $25.6 \pm \mathbf{6 . 9 1}$ & $\mathbf{2 1 . 8} \pm \mathbf{2 . 6 8}$ \\
Height $(\mathbf{c m})$ & $\mathbf{1 7 1 . 4} \pm 9.10$ & $\mathbf{1 6 8 . 6} \pm \mathbf{3 . 8 5}$ \\
Mass $(\mathbf{k g})$ & $\mathbf{8 5 . 2} \pm \mathbf{2 2 . 7 5}$ & $\mathbf{6 8 . 2} \pm \mathbf{4 . 0 9}$ \\
Body Mass Index $\left(\mathbf{k g} / \mathbf{m}^{2}\right)$ & $29.0 \pm 7.08$ & $\mathbf{2 3 . 9} \pm \mathbf{1 . 1 9}$ \\
\hline
\end{tabular}

between 18 and 30 years, completed a physical activity readiness questionnaire (PARQ) and informed consent, and were accepted for inclusion if they had no signs or symptoms of disease, no orthopaedic injuries, and confirmed via self-report that they were not using any medication or performance enhancing substances which might affect the study. Participants were instructed to maintain their current exercise and nutritional habits but to avoid engaging in any lower body resistance training for 4 weeks prior to, and throughout the duration of the experiment. Due to the duration of the intervention, participants were required to attend $100 \%$ of the training sessions.

\section{Strength Testing}

Lower-body maximum strength was assessed by unilateral 1-repetition maximum (RM) testing on the leg press, knee extension and seated knee flexion exercises (Nautilus Evo, Nautilus, Vancouver, Washington, US). Prior to testing all participants attended a familiarization session where they were given clear instructions and demonstrations on how to perform all the exercises correctly, seat positions were determined, range of motion assessed, and all details recorded for use throughout testing and training. During familiarization, participants performed two sets of each exercise bilaterally, using a load equating to an estimated $50 \%$ of $1 \mathrm{RM}$ for 6-10 repetitions. For the purposes of testing and training full range of motion was determined as the following: leg press - from full hip flexion (the point immediately before the pelvis rotates anteriorly) to near full knee extension (i.e. just before lockout), knee extension - from full flexion to full knee extension, seated knee flexion - from full knee extension to complete knee flexion. For pre-intervention testing participants reported to the laboratory having refrained from any exercise other than activities of daily living for at least 48 hours prior. Participants were required to perform a specific warm up focusing on each of the lower limb movements for familiarization, to raise core temperature and facilitate neuromuscular rehearsal of the specific unilateral exercise. ${ }^{26}$ Participants performed a general warm-up, completing two sets of bilateral leg press exercise, using a load equating to an estimated $50 \%$ of 1RM for 6-10 repetitions. Specific warm-up and 1RM testing was completed in the following order; dominant followed by non-dominant leg for leg press, knee extension and seated knee flexion. Participants selected a load based on the warm up estimated to be $\sim 80 \%$ of estimated $1 \mathrm{RM}$ and performed $\sim 3$ unilateral repetitions. Following a 2-minute rest, participants then attempted an estimated 1RM and increased the weight by small increments of 5-10\% after each successful attempt until their 1RM was attained. Each maximal attempt was separated with a 3-5 minute rest. The 1RM was obtained within 3 attempts for each participant. Participants rested for 5 minutes before repeating the specific warm-up and maximal testing protocol for the non-dominant leg and between exercises. This process was repeated for postintervention testing, with at least 72 hours rest between the final training session and the post-intervention testing.

\section{Training}

A one-to-one supervised unilateral RT intervention was performed $\left(2 \mathrm{~d}^{-}\right.$week $\left.^{-1}\right)$ with at least 72 hours between sessions. The intervention lasted for 6 weeks using a load of $70 \%$ of $1 \mathrm{RM}$ and was performed on the same resistance machines used for testing. The order of lower limbs trained was alternated each session (in sessions 1, 3, 5, 7, 9 and 11 the MJ leg was trained first, followed by the SJ leg, and vice versa for sessions 2, 4, 6, 8, 10 and 12), this also allowed for alternation of training the dominant/non-dominant leg first in each session. Participants performed 3 sets of each exercise, to momentary failure using a 2 second concentric: 2 second eccentric repetition duration. This meant that volume with respect to exercises was not maintained between SJ and MJ limbs; instead the MJ limb performed 3 total sets of exercise whilst the contralateral SJ limb performed 6 total sets ( 3 each of knee extension and seated knee flexion) to volume match the exercise with respect to muscle group. A rest interval of 3 minutes was provided between sets and exercises. If a participant performed $>15$ repetitions in a set then the load was increased by 5\% for successive sets in the same session, if they performed 


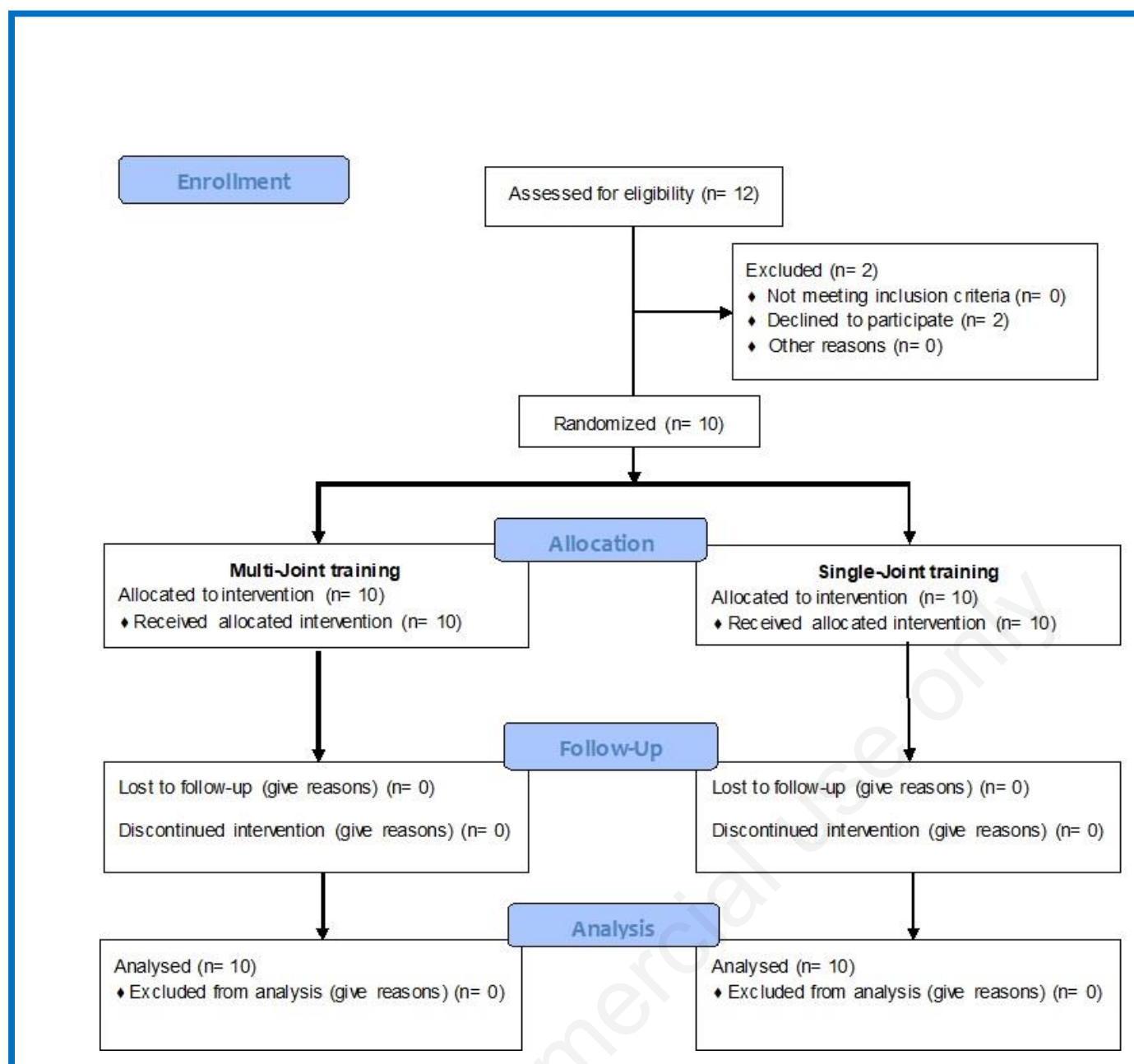

Fig 1. CONSORT diagram showing enrolment, allocation, continuance and analysis.

$<6$ repetitions the load was decreased by $5 \%$ for the successive sets. If a participant performed $>8$ repetitions for each of the 3 sets for a given leg for an entire workout then the load was increased by $5 \%$ for the start of the next workout.

\section{Statistical analysis}

Figure 1 is the CONSORT diagram showing enrolment, allocation, continuance, and analysis. Since participants were in both groups the participant numbers reflect this throughout the flow-diagram. Due to the hierarchical nature of the data multilevel mixed modelling was used. The hierarchical structure of the data included participants as a level 2 variable, and condition as the level 1 variable. The effects of the independent variable 'condition' (i.e. MJ or SJ) upon the dependent variables of change in 1RM (i.e. post- minus pre-scores) for leg press, knee extension, and seated knee flexion was examined. 'Condition' was modelled as a fixed effect with random intercepts by participant included, and baseline 1RM values (i.e. pre-score for 1RM leg press, knee extension, and knee flexion respectively) were included as fixed covariates. A variance components covariance type was used and Restricted Maximum Likelihood estimation. Adjusted estimated marginal means for changes in 1RM and 95\% confidence intervals were calculated and presented. As there were only 2 conditions paired comparisons for estimations of fixed effects are reported from the model. All analyses were performed using SPSS (version 24; IBM Corp, Portsmouth, Hampshire, UK) and $p \leq 0.05$ set as the limit for statistical significance. Paired comparison estimation plots were also produced using Estimation Statistics ${ }^{27}$ for data visualization of the dependent variables (i.e. change in $1 \mathrm{RM}$ ) in each condition. These were to show individual data along with the paired delta and 95\% confidence intervals for the delta for changes in 1RM. All results are reported in the units of measurement for each test.

\section{Results}

Multilevel modelling resulted in a significant effect by 'condition' for change in $1 \mathrm{RM}$ leg press $\left(t_{(9.479)}=5.150\right.$, $p=0.001$; mean difference $[95 \%$ CIs] $=7.3$ [4.1 to 10.5]), 
Single- versus multi-joint lower body resistance training

Eur J Transl Myol 29 (1): 14-21, 2019

Table 2. Pre-intervention results, post-intervention results, and changes in strength

\begin{tabular}{|c|c|c|c|}
\hline \multirow[t]{2}{*}{ Variable } & Pre-intervention & Pre-intervention & Changes \\
\hline & [95\%CIs] & [95\% CIs] & $\begin{array}{c}\text { Marginal Means } \\
\text { [95\%CIs]) }\end{array}$ \\
\hline \multicolumn{4}{|l|}{$\begin{array}{l}\text { Leg Press 1RM } \\
\text { (kg) }\end{array}$} \\
\hline MJ & $\begin{array}{c}122.1 \pm 40.2 \\
{[93.3 \text { to } 150.9]}\end{array}$ & $\begin{array}{c}170.1 \pm 34.5 \\
{[145.4 \text { to } 194.8]}\end{array}$ & $\begin{array}{c}48.0[42.1 \text { to } \\
53.8]\end{array}$ \\
\hline SJ & $\begin{array}{c}122.6 \pm 38.1 \\
{[95.4 \text { to } 150.0]}\end{array}$ & $\begin{array}{c}163.2 \pm 35.4 \\
{[137.9 \text { to } 188.5]}\end{array}$ & $\begin{array}{c}40.6[34.8 \text { to } \\
46.5]\end{array}$ \\
\hline \multicolumn{4}{|l|}{$\begin{array}{l}\text { Knee Ext. 1RM } \\
\text { (kg) }\end{array}$} \\
\hline MJ & $\begin{array}{c}57.2 \pm 11.1 \\
{[49.3 \text { to } 65.1]}\end{array}$ & $\begin{array}{c}80.8 \pm 13.1 \\
{[71.4 \text { to } 90.2]}\end{array}$ & $\begin{array}{c}23.6[21.9 \text { to } \\
25.2]\end{array}$ \\
\hline SJ & $\begin{array}{c}56.9 \pm 10.0 \\
{[49.7 \text { to } 64.1]}\end{array}$ & $\begin{array}{c}76.0 \pm 11.5 \\
{[67.8 \text { to } 84.2]}\end{array}$ & $\begin{array}{c}19.1[17.5 \text { to } \\
20.8]\end{array}$ \\
\hline \multicolumn{4}{|l|}{$\begin{array}{l}\text { Knee Flex. } \\
1 R M(k g)\end{array}$} \\
\hline MJ & $\begin{array}{c}35.9 \pm 11.3 \\
{[27.9 \text { to } 44.0]}\end{array}$ & $\begin{array}{c}60.2 \pm 12.3 \\
{[51.4 \text { to } 69.0]}\end{array}$ & $\begin{array}{c}24.3[21.6 \text { to } \\
27.0]\end{array}$ \\
\hline SJ & $\begin{array}{c}36.7 \pm 10.7 \\
{[29.1 \text { to } 44.4]}\end{array}$ & $\begin{array}{c}55.7 \pm 11.4 \\
{[47.6 \text { to } 63.8]}\end{array}$ & $\begin{array}{c}19.0[16.3 \text { to } \\
21.7]\end{array}$ \\
\hline
\end{tabular}

knee extension $\left(t_{(9.597)}=7.278, p \leq 0.001\right.$; mean difference $[95 \% \mathrm{CIs}]=4.5$ [3.1 to 5.8]), and knee flexion $\left(t_{(9.679)}=6.119, p \leq 0.001\right.$; mean difference [95\%CIs] $=$ 5.3 [3.3 to 7.2]. All dependent variables were significantly greater for the MJ group compared with the SJ group. Table 2 reports pre- and post-intervention $1 \mathrm{RMs}$ for each exercise, in addition to unadjusted and adjusted means, and adjusted $95 \%$ CIs. Inspection of the 95\% CIs suggested that both conditions resulted in significant changes in 1RM strength for all exercises. Figure 2 shows paired comparison estimation plots for each exercise for the changes in 1RM.

\section{Discussion}

The aims of the present study were to compare the strength adaptations following unilateral $\mathrm{MJ}$ and SJ resistance training in the lower limbs in recreationally active males and females. This study adds to the body of literature comparing SJ and $\mathrm{MJ}$ research in the lower limbs, whilst also considering both male and female participants, the latter of which is lacking in RT research. Our analyses revealed that both groups showed increases in maximal strength (1RM) for all tested exercises (LP, $\mathrm{KE}$ and $\mathrm{KF}$ ) that we would consider meaningful with precise estimates, in addition to being statistically significant based upon within condition 95\% CIs (see table 2). However, the MJ group, performing only LP, produced greater strength increases for all exercises compared to the SJ group (which performed both KE and KF exercises) all of which were statistically significant and estimated with good precision based upon the 95\% CIs. This data is similar to that reported in a previous study considering amateur male soccer players, ${ }^{13}$ which showed greater increases in back squat and knee extension 1RM for persons training using MJ- compared to those training using SJ-exercises. In contrast, this data somewhat conflicts with other research which has suggested similar strength adaptations comparing MJ vs. $\mathrm{SJ}^{12}$ as well as MJ vs. MJ+SJ exercise groups. ${ }^{9,10}$ However, these studies all considered only upper body exercises for both training and testing, and none involved the knee flexors. Perhaps most interestingly, the present study seems to challenge our understanding of training specificity and development of motor schema. Evidence has suggested that exercising using a specific exercise, and using specific loads, serves as practice, and thus reinforces the motor schema required for that movement and load. ${ }^{14}$ In this sense, many persons might perform a variety of exercises under the pretence of needing to do so to increase strength and muscle size across each of those exercises. However, the present study suggests that a general strength increase is possible which might 

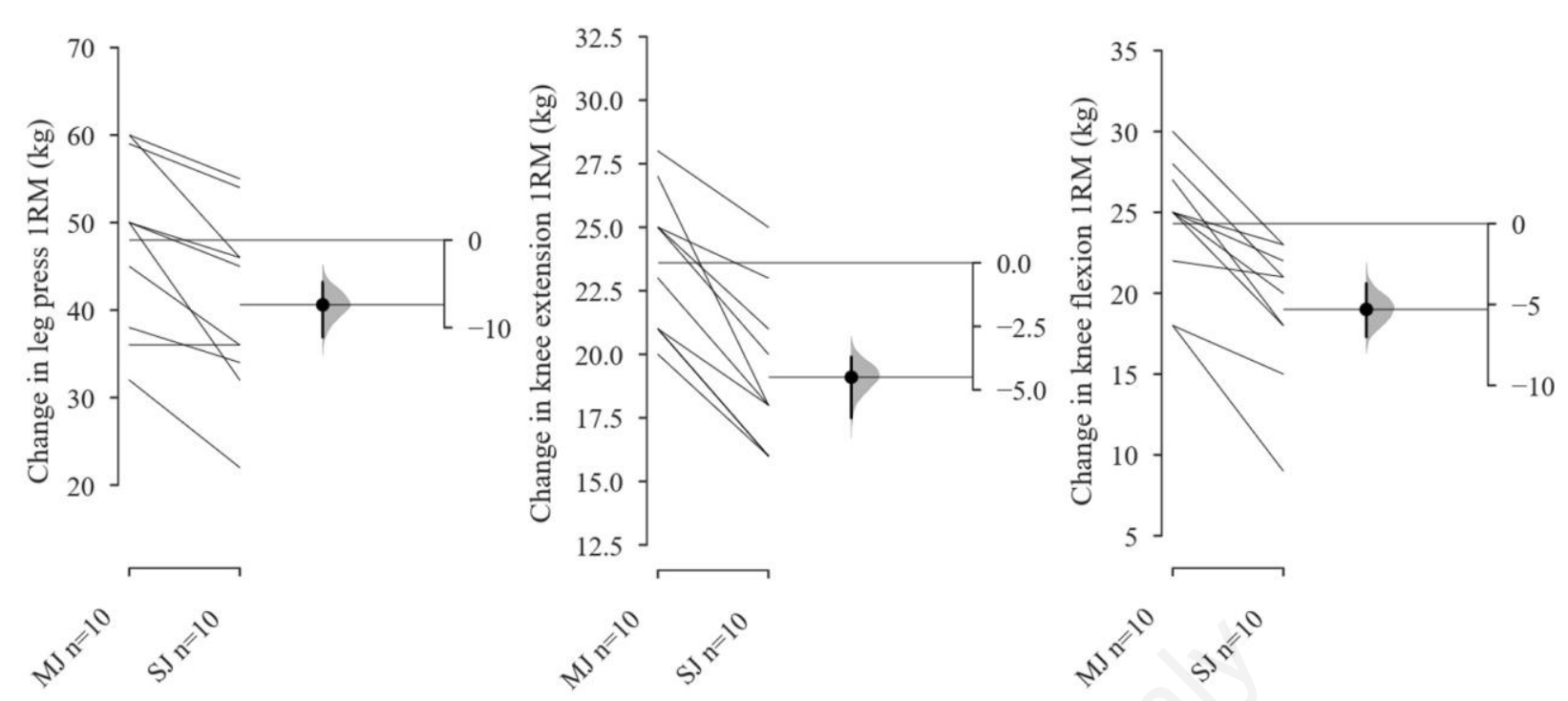

Fig 2. Paired comparison estimation plots for each exercise for the changes in 1RM.

provide transference to other exercises and potentially other movements. We propose that this is often a more desirable outcome in resistance training; not the improvement in strength of specific exercises/ movements but rather improved functioning across multiple movements, as well as in daily life, by a more general increase in strength. In the present study, strengthening the muscles of the lower body using only a MJ exercise resulted in additional strength increases in both $\mathrm{KE}$ and $\mathrm{KF}$ (SJ) exercises compared with only performing these $\mathrm{SJ}$ exercises. Considering the results of the present study in addition to those of Paoli et al. ${ }^{13}$, it seems there may be greater strength gains in the lower body for MJ exercises. However, these differences may be small and potentially not meaningful (point estimates in the present study ranging from $4.5 \mathrm{~kg}$ to $7.3 \mathrm{~kg}$ for mean differences). Although the MJ intervention was more time efficient and seems to produce at least similar changes compared to SJ only exercises. Since time constraints and perceived difficulty are often cited barriers to exercise ${ }^{28}$ it is important to find ways to improve time efficiency and simplicity which might serve to promote greater adherence. Future research should consider how the use of RT programmes using SJ and $\mathrm{MJ}$ exercises translates in to performance of realworld activities (such as walking up a flight of stairs, hiking, etc.), sporting movements (such as change of direction, vertical jump, etc.) and/or functional movements (such as timed up-and-go, sit-to-stand tasks, etc.). In addition to the time efficient nature of MJ exercise from a practical perspective, our results might also have potential applications for injury prevention strategies. Knee flexor strengthening has been a specific aim for injury treatment and prevention, ${ }^{16,17}$ yet as noted there is a lack of studies examining knee flexor strength changes resulting from MJ exercise. Knee flexion strength is associated with injury risk ${ }^{4,5}$ though knee flexor strengthening is usually performed using SJ exercises. ${ }^{16}$ This may be based upon the assumption that the hamstrings are not adequately strengthened during lower-body MJ exercises such as squats and leg press, since it concomitantly shortens in the knee while lengthens in the hip joint. Indeed, previous studies show that the electromyographical amplitude of the hamstrings are limited in lower body MJ exercises. ${ }^{20,21}$ However, our results do not support this and instead suggest that it may be possible to increase knee flexor strength using MJ exercise alone. Future research might consider comparison of time efficient MJ interventions to SJ interventions for increasing knee flexor strength and reducing injury risk. Further practical implications are suggested by considering our findings in addition to previous research from Soares, et al. ${ }^{15}$ Their findings suggested greater fatigue immediately- and 24 hourspost exercise resulting from SJ compared to MJ exercise. As such, it might be that performing only MJ exercises in a RT protocol could prevent unnecessary fatigue and soreness combined with greater strength increases. However, the study by Soares, et al. ${ }^{15}$, only considered upper body exercises, and so future research should consider fatigue decrement and DOMS following SJ and MJ exercise protocols using lower-body exercises. Finally, it is important to address the limitations of the present paper. It could be argued that the lack of morphological measurements of muscle thickness or muscle fibre-size change is a limitation, as well as the duration of the intervention. Indeed, there may have been differences between the $\mathrm{MJ}$ and $\mathrm{SJ}$ interventions examined in the present study with respect to these outcomes. Future studies might consider longitudinal 
designs where variation is provided following an initial SJ or MJ intervention. However, at present, our evidence suggests that for those looking to increase strength the use of a MJ exercise is sufficient. Since our results do contradict some previous research considering SJ and MJ exercise, it is worth noting the differences in research design. For example, previous studies assessed strength increases using isokinetic dynamometry to report peak torque, rather than 1RM testing as used herein ${ }^{9,15}$ and it has been suggested that isokinetic dynamometry and $1 \mathrm{RM}$ testing methods produce conflicting results for assessing strength. $^{29}$ Furthermore, we should also consider the greater heterogeneity in adaptation between, as opposed to within-participants, which was controlled by our unilateral study design. This also enabled us to utilise a multilevel approach to data analysis.

The present study adds to the dearth of literature considering female participants in addition to males, as well as that comparing SJ and MJ lower-body exercises using a within participant design. The data suggest that performing only lower body MJ exercise may produce greater strength increases for both MJ and SJ movements than training using SJ exercises, though the differences are small. However, considering the time efficiency of the MJ intervention, this research serves to support the use of low-volume, minimal $\operatorname{dose}^{30} \mathrm{MJ}$ resistance training protocols which might serve to promote greater adherence. Furthermore, the data presented might be useful to strength and conditioning coaches in strengthening with the aim of injury risk reduction and their management of RT volume in athletes, where fatigue is a key factor.

\section{List of acronyms}

CI - Confidence Intervals

DOMS - Delayed Onset Muscle Soreness

$\mathrm{KE}$ - Knee Extension

$\mathrm{KF}$ - Knee Flexion

MJ - Mulit-joint

RM - Repetition Maximum

RT - Resistance Training

SJ - Single Joint

\section{Authors contributions}

All named authors played a substantial role in the conception and/or study design, data acquisition and/or analysis, as well as drafting of the manuscript, approval of the final version to be published, and agree the accountability for all aspects of accuracy and integrity of the work.

\section{Acknowledgments None.}

Funding No funding was obtained for this research project.

\section{Conflict of Interest}

The authors declare they have no financial, personal, or other conflicts of interest..

\section{Ethical Publication Statement}

We confirm that we have read the Journal's position on issues involved in ethical publication and affirm that this report is consistent with those guidelines.

\section{Corresponding Author}

James Peter Fisher, School of Sport, Health and Social Science, Southampton Solent University, East Park Terrace, Southampton, UK. Tel: +44 2382013163

Email: james.fisher@solent.ac.uk

\section{E-mails of co-authors}

Alexander Goncalves: sachagoncalves@outlook.com

Paulo Gentil: paulogentil@hotmail.com

James Steele: James.steele@solent.ac.uk

Jürgen Giessing: giessing@uni-landau.de

Antonio Paoli: antonio.paoli@unipd.it

\section{References}

1. Srikanthan P, Karlamangla, AS. Muscle mass index as a predictor of longevity in older adults. Am J Med 2014;127(6):547-53.

2. Ruiz JR, Sui X, Lobelo F, et al. Association between muscular strength and mortality in men: Prospective cohort study. Br Med J 2008;337:a439.

3. Steele J, Fisher J, Skivington M, et al. A higher effort-based paradigm in physical activity and exercise for public health: Making the case for a greater emphasis on resistance training. BMC Public Health 2017;17(1):300.

4. de la Motte SJ, Gribbin TC, Lisman P, et al. Systematic review of the association between physical fitness and musculoskeletal injury risk: Part 2-Muscular endurance and muscular strength. J Strength Cond Res 2017;31(11):3218-34.

5. Opar DA, Serpell BG. Is there a potential relationship between prior hamstring strain injury and increased risk for future anterior cruciate ligament injury? Arch Phys Med Rehab 2014;95:401-5.

6. Arikawa AY, O'Dougherty M, Schmitz KH. Adherence to a strength training intervention in adult women. J Phys Act Health 2011;8:111-8.

7. Westcott WL, Winett RA, Annesi JJ, et al. Prescribing physical activity: Applying the ACSM protocols for exercise type, intensity, and duration across 3 training frequencies. Physician. Sportsmedicine 2009;37:51-58.

8. Fisher J, Steele J, McKinnon P, and McKinnon, S. Strength gains as a result of brief, infrequent resistance exercise in older adults. J Sports Med 2014; Article ID 731890.

9. Gentil P, Soares SRS, Pereira MC, et al. Effect of adding single-joint exercises to a multi-joint exercise resistance-training program on strength and hypertrophy in untrained subjects. Appl Phys Nutr Metab 2013;38:341-4. 
10. de França HS, Branco PAN, Guedes Junior DP, et al. The effects of adding single-joint exercises to a multi-joint exercise resistance training program on upper body muscle strength and size in trained men. Appl Phys Nutr Metab 2015;40:822-6.

11. Barbalho M, Gentil P, Raiol R, et al. Influence of adding single-joint exercise to a multijoint resistance training program in untrained young women. J Strength Cond Res 2018;E-Pub ahead of print.

12. Gentil P, Soares S, Bottaro M. Single vs. multi-joint resistance exercises: Effects on muscle strength and hypertrophy. Asian J Sports Med 2015;6:e24057.

13. Paoli A, Gentil P, Moro T, et al. Resistance training with single vs. multi-joint exercises at equal total load volume: Effects on body composition, cardiorespiratory fitness, and muscle strength. Front Physiol 2017;8:1105.

14. Fisher J, Steele J, Smith D. High-and low-load resistance training: Interpretation and practical application of current research findings. Sports Me. 2017;47:393-400.

15. Soares S, Ferreira-Junior JB, Pereira MC, et al. Dissociated time course of muscle damage recovery between single-and multi-joint exercises in highly resistance-trained men. J Strength Cond Res 2015;29:2594-9.

16. Ter Stege MH, Dallinga JM, Benjaminse A, Lemmink KA. Effect of interventions on potential, modifiable risk factors for knee injury in team ball sports: A systematic review. Sports Med 2014;44:1403-26.

17. Hughes G. A review of recent perspectives on biomechanical risk factors associated with anterior cruciate ligament injury. Res Sports Med 2014;22:193-212.

18. Holm I, Risberg MA, Aune AK, et al. Muscle strength recovery following anterior cruciate ligament reconstruction: A prospective study of 151 patients with a two-year follow-up. Isokinetics Exerc Sci 2000;8:57-63.

19. Øiestad BE, Holm I, Aune AK, et al. Knee function and prevalence of knee osteoarthritis after anterior cruciate ligament reconstruction: A prospective study with 10 to 15 years of follow-up. Am J Sports Med 2010;38:2201-10.

20. Escamilla RF, Fleisig GS, Zheng N, et al. Biomechanics of the knee during closed kinetic chain and open kinetic chain exercises. Med Sci Sports Exerc 1998;30:556-9.

21. Wilk KE, Escamilla RF, Fleisig GS, et al. A comparison of tibiofemoral joint forces and electromyographic activit during open and closed kinetic chain exercises. Am J Sports Med 1996;24:518-27.

22. MacInnis MJ, McGlory C, Gibala MJ, Phillips SM. Investigating human skeletal muscle physiology with unilateral exercise models: When one limb is more powerful than two. Appl Phys Nutr Metab 2017;42:563-70.

23. Fisher J, Blossom D, Steele J. A comparison of volume equated knee extensions to failure or not to failure upon rating of perceived exertion and strength adaptations. Appl Phys Nutr Metab 2016;41:168-74.

24. Fisher J, Ironside M, Steele J. Heavier- and lighterload resistance training to momentary failure produce similar increases in strength with differing degrees of discomfort. Muscle Nerve 2017;56:797803.

25. Cumming G. ESCI 2001 to 2010. Retrieved from https://thenewstatistics.com/itns/esci/esci-2001-to2010/.

26. Shellock FG, Prentice WE. Warming-up and stretching for improved physical performance and prevention of sports-related injuries. Sports Med 1985;2:267-78.

27. Ho J, Claridge-Chang A. Estimation statistics. Retrieved from http://www.estimationstats.com

28. Trost SG, Owen N, Bauman AE, et al. Correlates of adults' participation in physical activity: Review and update. Med Sci Sports Exerc 2002;34:19962001.

29. Gentil P, Del Vecchio FB, Paoli A, et al. Isokinetic dynamometry and 1RM tests produce conflicting results for assessing alterations in muscle strength. J Human Kinetics 2017;56:19-27.

30. Fisher JP, Steele J, Gentil P, et al. A minimal dose approach to resistance training for the older adult; the prophylactic for aging. Exp Gerontol 2017;99:80-6.

Submissions: January, 25, 2019

Revision received: February 18, 2019

Acceptance: February 18, 2019 